\title{
Dominant antibody responses to Fuca1-3GalNAc and Fucal-2Fucal-3GlcNAc containing carbohydrate epitopes in Pan troglodytes vaccinated and infected with Schistosoma mansoni ${ }^{\text {is }}$
}

\author{
A. van Remoortere, ${ }^{\mathrm{a}, 1}$ H.J. Vermeer, ${ }^{\mathrm{a}, 1}$ A.M. van Roon, ${ }^{\mathrm{a}}$ J.A. Langermans, ${ }^{\mathrm{b}}$ \\ A.W. Thomas, ${ }^{\mathrm{b}}$ R.A. Wilson, ${ }^{\mathrm{c}}$ I. van Die, ${ }^{\mathrm{d}}$ D.H. van den Eijnden, ${ }^{\mathrm{d}}$ K. Ágoston, ${ }^{\mathrm{e}}$ \\ J. Kérèkgyarto, ${ }^{\mathrm{e}}$ J.F.G. Vliegenthart, ${ }^{\mathrm{f}}$ J.P. Kamerling, ${ }^{\mathrm{f}}$ G.J. van Dam, ${ }^{\mathrm{a}}$ C.H. Hokke, ${ }^{\mathrm{a}, *}$ \\ and A.M. Deelder ${ }^{\mathrm{a}}$ \\ a Department of Parasitology, Leiden University Medical Center, P.O. Box 9600, NL-2300 RC, Leiden, The Netherlands \\ ${ }^{\mathrm{b}}$ Department of Parasitology, Biomedical Primate Center, P.O. Box 3306, NL-2280 GH, Rijswijk, The Netherlands \\ ${ }^{\mathrm{c}}$ Department of Biology, University of York, P.O. Box 373, York YO10 5 YW, England, UK \\ ${ }^{\mathrm{d}}$ Department of Molecular Cell Biology, VU University Medical Center, Van der Boechorststraat 7, NL-1081 BT, Amsterdam, The Netherlands \\ ${ }^{\mathrm{e}}$ Institute of Biochemistry, Faculty of Sciences, University of Debrecen, P. O. Box 55, H-4010, Debrecen, Hungary \\ ${ }^{f}$ Bijvoet Center, Department of Bio-Organic Chemistry, Utrecht University, P.O. Box 80.075, NL-3508 TB, Utrecht, The Netherlands
}

Received 5 May 2003; received in revised form 5 November 2003; accepted 11 December 2003

\begin{abstract}
The development of the humoral anti-glycan immune response of chimpanzees, either or not vaccinated with radiation-attenuated Schistosoma mansoni cercariae, was followed during 1 year after infection with $S$. mansoni. During the acute phase of infection both the vaccinated and the control chimpanzees produce high levels of immunoglobulin $\mathrm{G}(\mathrm{IgG})$ antibodies against carbohydrate structures that are characteristic for schistosomes carrying the Fuc 1 1-3GalNAc and Fuc 1 1-2Fuc $\alpha 1-3$ GlcNAc motifs, but not to the more widespread occurring structures GalNAc $\beta 1-4 G l c N A c$, GalNAc $\beta 1-4($ Fuc $\alpha 1-3)$ GlcNAc, and Gal $\beta 1-4($ Fuc $\alpha 1-3)$ GlcNAc (Lewis $\left.{ }^{\mathrm{x}}\right)$. In addition, high levels of $\operatorname{IgM}$ antibodies were found against the trimeric Lewis ${ }^{\mathrm{x}}$ epitope. Apparently, the schistosome-characteristic carbohydrate structures are dominant epitopes in the anti-glycan humoral immune response of the chimpanzees. All chimpanzees showed an increase in the level of antibodies against most of the carbohydrate structures tested directly after vaccination, peaking at challenge time and during the acute phase of infection. With the exception of anti-F-LDN antibody responses, the anti-carbohydrate antibody responses upon schistosome infection of the vaccinated animals were muted in comparison to the control animals.

(C) 2003 Elsevier Inc. All rights reserved.

Index Descriptors and Abbreviations: Anti-carbohydrate antibodies, glycoconjugates, humoral immune response, infection, Schistosoma mansoni, synthetic glycan epitopes, trematode, vaccination, irradiated cercariae. Circulating cathodic antigen (CCA), Fuc $\alpha 1-3 \mathrm{GalNAc}(\mathrm{Fuc} \alpha 1-3)_{0 / 1}$ GlcNAcaGal (F-LDN(-F) $)_{0 / 1}$ ), goat anti-human IgG (GaHuIgG), goat anti-human IgM (GaHuIgM), Hepes-buffered saline (HBS), immunoglobulin (Ig), keyhole limpet haemocyanin (KLH), GalNAc $\beta 1-4 G l c N A c$ (LDN), GalNAc $\beta 1-4(F u c \alpha 1-3)$ GlcNAc (LDN-F), GalNAc $\beta 1-4(F u c \alpha 1-2 F u c \alpha 1-$ 3)GlcNAc (LDN-DF), Galß1-4(Fuc 1 1-3)GlcNAc (Lewis ${ }^{\mathrm{x}}$ ), peripheral blood mononuclear cells (PBMCs), response units (RUs), surface plasmon resonance (SPR).
\end{abstract}

All procedures were approved by independent Scientific and Ethical Committees, and were performed following the recommendations and guidelines of the Primate Vaccine Evaluation Network of the European Commission.

* Corresponding author. Fax: +31-71-526-69-07.

E-mail address: c.h.hokke@lumc.nl (C.H. Hokke).

${ }^{1}$ Both authors contributed equally to this article.

\section{Introduction}

Infection of the definitive host with the trematode parasite Schistosoma mansoni gives rise to both humoral and cell-mediated immune responses. The humoral immune response is directed primarily against schistosomal glycoconjugates (Omer-Ali et al., 1989), which are thought to be directly involved in many aspects of the 
parasite-host interaction (Cummings and Nyame, 1999; Hokke and Deelder, 2001). Various developmental stages of schistosomes express a wide variety of glycoconjugates. The carbohydrate elements carried by these glycoconjugates may be identical or similar to endogenous glycans occurring in the mammalian hosts, while other structural elements occur that are non-mammalian and may be characteristic for schistosomes. Some glycoconjugates, such as several schistosome circulating antigens are presented or excreted in relatively large quantities (Berggren and Weller, 1967; Deelder et al., 1980). The immunogenic glycan part of the circulating anodic antigen is a unique polysaccharide consisting of repetitive disaccharide units of GlcA $\beta 1-3$ GalNAc (Bergwerff et al., 1994), and the major glycans of the circulating cathodic antigen (CCA) contain repeating units of Galß1-4(Fuc $\alpha 1-3)$ GlcNAc (Lewis ${ }^{\mathrm{x}}$ ) (Van Dam et al., 1994). In two life-cycle stages, the infectious cercariae and the pathology-causing eggs, multi-fucosylated structures containing (Fuc $\alpha 1-2)_{0 / 1}$ Fuc $\alpha 1-2 F u c \alpha 1-$ $3 \mathrm{GlcNAc}$ sequences with terminal Fuc $\alpha 1-3 \mathrm{GalNAc}$, have been described (Khoo et al., 1997a,b, 1995; Wuhrer et al., 2002). So far, these structures have not been found in mammals or other higher organisms.

In addition, monomeric Lewis ${ }^{\mathrm{x}}$, GalNAc $\beta 1-4 \mathrm{Glc}-$ NAc (LacdiNAc, LDN) and GalNAc $\beta 1-4$ (Fuc $\alpha 1-$ 3)GlcNAc (LDN-F) epitopes were found in various life-cycle stages of schistosomes (Ko et al., 1990; Van Remoortere et al., 2000). These epitopes are not only found for schistosomes but are shared with the host (Bergwerff et al., 1995; Dell et al., 1995; Fox et al., 1983; Fukuda et al., 1985; Hakomori et al., 1981; Morris et al., 1996; Spooncer et al., 1984; Van den Eijnden et al., 1995; Van Kuik et al., 1991). Recently, we demonstrated that sera of Schistosoma-infected individuals contain high levels of antibodies against the schistosome-characteristic GalNAc $\beta 1-4$ (Fuc $\alpha 1-2$ Fuc $\alpha 1-$ 3)GlcNAc (LDN-DF) and Fuc $\alpha 1-3$ GalNAc $\beta 1-4 G l c-$ NAc (F-LDN) epitope in comparison with LDN, LDN-F, and Lewis ${ }^{\mathrm{x}}$ epitopes (Naus et al., 2003; Van Remoortere et al., 2001). In an earlier study, it was shown that chimpanzees vaccinated by multiple exposures to radiation-attenuated cercariae (RA) developed a strong cellular and humoral immune response and showed a $40 \%$ reduction in infection intensity compared to infected control chimpanzees (Eberl et al., 2001a). In both groups the humoral immune response during the acute phase of infection was predominantly directed against glycans associated with cercariae and eggs. The glycan epitopes recognized included LDN, LDN-F, Lewis ${ }^{\mathrm{x}}$, and undefined glycans present on keyhole limpet haemocyanin (KLH). It was suggested that the intense and early anti-glycan response to larvae and eggs could act as a smokescreen to divert the immune system away from peptide epitopes present on the more vulnerable larvae (Eberl et al., 2001b).
In this study we have evaluated the anti-carbohydrate antibody response of the $S$. mansoni-infected chimpanzees, both RA-vaccinated and non-vaccinated using the same sera as Eberl et al. (2001b). We have focused on the response against schistosome-characteristic epitopes (e.g., LDN-DF, Fuc $\alpha 1-3 \mathrm{GalNAc}$ (Fuc $\alpha 1$ $3)_{0 / 1}$ GlcNAc $\alpha$ Gal (F-LDN-F $\left.0 / 1\right)$ ), using a set of synthetic glycoconjugates in combination with surface plasmon resonance (SPR) as analysis technique, which was previously shown to be well-suited for determining antibody responses, especially when antigen availability is limited (Van Remoortere et al., 2001). In addition, we compared the antibody responses against the host-shared epitopes (e.g., LDN, LDN-F, and Lewis ${ }^{\mathrm{x}}$ ) to those that were reported previously (Eberl et al., 2001b).

\section{Materials and methods}

\subsection{Sera of chimpanzees}

The vaccination trial was carried out in six unrelated healthy 5-6 year old male chimpanzees (Pan troglodytes) as recently described in detail (Eberl et al., 2001a). Briefly, three chimpanzees (V1, 2, 3) were exposed three times to 9000 attenuated $S$. mansoni cercariae (300 Gy of $\gamma$-radiation from ${ }^{60} \mathrm{Co}$ source) at 5 -week intervals. Three weeks after the last exposure, these chimpanzees and three control chimpanzees $(\mathrm{C} 1,2,3)$ were challenged percutaneously with 2000 normal $S$. mansoni cercariae. At the end of the study, at time points between postchallenge (p.c.) weeks 28 and 36, all chimpanzees were cured with Praziquantel (Droncit; Bayer) until proven to be egg-negative. Sera were taken from each chimpanzee at 1-3-weeks interval (Eberl et al., 2001a) and stored at $-80^{\circ} \mathrm{C}$ until further use. In our studies we used the same serum samples as described in the study of Eberl et al. (2001a).

\subsection{Synthetic antigens}

GalNAc $\beta 1-4 G l c N A c \beta 1-3 G a l \alpha 1$ (LDN), Fuc $\alpha 1-3 G a l$ NAc $\beta 1-4 G l c N A c \beta 1-3 G a l \alpha 1 \quad$ (F-LDN), GalNAc $\beta 1-4$ (Fuc $\alpha 1-3)$ GlcNAc $\beta 1-3 G a l \alpha 1$ (LDN-F), Fuc $\alpha 1-3 \mathrm{Gal}$ NAc $\beta 1-4($ Fuc $\alpha 1-3)$ GlcNAc $\beta 1-3 G a l \alpha 1$ (F-LDN-F), and GalNAc $\beta 1-4$ (Fuc $\alpha 1-2$ Fuc $\alpha 1-3)$ GlcNAc (LDN-DF) were (enzymatically) synthesized and conjugated to bovine serum albumin (BSA) as described elsewhere (Ágoston et al., 2002; Halkes et al., 1998; Van Remoortere et al., 2000). Mono-, di-, and trimeric Lewis ${ }^{\mathrm{x}}$ human serum albumin (HSA) conjugates were a kind gift from Dr. B. Appelmelk (VUMC, Amsterdam). An overview of the synthesized glycan epitopes and their occurrences in various stages of the schistosome is given in Table 1 . 
Table 1

An overview of the synthesized glycan structures with their abbreviations

\begin{tabular}{|c|c|c|c|}
\hline & Carbohydrate structures & Occurrence by chemical characterization ${ }^{\mathrm{a}}$ & Occurence by histochemistry ${ }^{\mathrm{b}}$ \\
\hline LDN & GalNAc $\beta 1-4$ GlcNAc $\beta 1-3$ Gal $\alpha 1$ & GCX & Cercs/Eggs/Adults \\
\hline LDN-F & GalNAc $\beta 1-4($ Fuc $\alpha 1-3)$ GlcNAc $\beta 1-3$ Gal $\alpha 1$ & GCX & Cercs/Eggs/Adults \\
\hline F-LDN & Fuc $\alpha 1-3$ GalNAc $\beta 1-4$ GlcNAc $\beta 1-3 G a 1 \alpha 1$ & GCX & Cercs/Eggs/Adults \\
\hline F-LDN-F & $\begin{array}{l}\text { Fuc } \alpha 1-3 \text { GalNAc } \beta 1-4(\text { Fuc } \alpha 1-3) \text { GlcNAc } \beta 1- \\
\text { 3Gal } \alpha 1\end{array}$ & $\mathrm{GCX}$ & Cercs \\
\hline LDN-DF & GalNAc $\beta 1-4[$ Fuc $\alpha 1-2$ Fuc $\alpha 1-3]$ GlcNAc $\beta 1$ & GCX/Eggs & Cercs/Eggs \\
\hline Lewis $^{x}$ & Gal $\beta 1-4($ Fuc $\alpha 1-3)$ GlcNAc $\beta 1$ & CCA/Eggs & CCA/Eggs/Adults \\
\hline $\begin{array}{l}\text { Dimeric } \\
\text { Lewis }^{\mathrm{x}}\end{array}$ & $-[3 \mathrm{Gal} \beta 1-4(\text { Fuc } \alpha 1-3) \text { GlcNAc } \beta 1]_{-2}$ & CCA & CCA/Eggs/Adults \\
\hline $\begin{array}{l}\text { Trimeric } \\
\text { Lewis }^{\mathrm{x}}\end{array}$ & $-[3 \mathrm{Gal} \beta 1-4(\mathrm{Fuc} \alpha 1-3) \mathrm{GlcNAc} \beta 1]_{-3}$ & CCA & CCA/Eggs/Adults \\
\hline
\end{tabular}

These structures are building blocks that might appear on different antigens expressed by various stages of the parasite.

GCX, glycocalyx of cercariae; Cercs, cercariae; Mir, miracidia; CCA circulating cathodic antigen.

${ }^{a}$ Characterized by structural analysis of the respective antigen preparations (Bergwerff et al., 1994; Khoo et al., 1997a,b, 1995; Van Dam et al., 1994; Wuhrer et al., 2002).

${ }^{\mathrm{b}}$ Characterized histochemically by defined MAbs on sections of the respective parasite stages (Deelder et al., 1996; Van Remoortere et al., 2000).

\subsection{SPR spectroscopy}

Surface plasmon resonance analysis was carried out using a BIAcore 3000 instrument (Biacore AB, Uppsala, Sweden). CM5 Sensor Chips, amino coupling kit and surfactant P-20 were also obtained from Biacore AB.

Binding studies were carried out according to Van Remoortere et al. (2001). Briefly, the neoglycoproteins were immobilized onto a CM5 sensorchip with similar density. All analyses were carried out at flow rates of $5 \mu \mathrm{l} / \mathrm{min}$ at $25^{\circ} \mathrm{C}$ using Hepes-buffered saline (HBS) buffer as an eluent. Sera were diluted 1:40 with running buffer containing $0.5 \% \mathrm{P}-20$. Injection times of sera were $2 \mathrm{~min}$ followed by $2 \mathrm{~min}$ of buffer injection to allow dissociation. The isotypes of the antibodies were determined subsequently by 2-min injection pulses with goat anti-human IgG (GaHuIgG) (Dako, Glostrup, Denmark) and goat anti-human IgM (GaHuIgM)(Dako), each followed by $2 \mathrm{~min}$ of dissociation time. Both antiisotype antibodies were diluted 1:100 with running buffer containing $0.5 \% \mathrm{P}-20$. After completion of each cycle, surfaces were regenerated with a 2-min injection pulse of $20 \mathrm{mM} \mathrm{HCl}$. To correct for refractive index changes and non-specific binding, BSA or HSA were used as blank.

\section{Results}

Anti-carbohydrate antibody responses from sera of vaccinated and infected chimpanzees were analyzed using surface plasmon resonance spectroscopy and a panel of neoglycoconjugates carrying defined glycan structures (Table 1). The binding profiles of antibodies directed against LDN and LDN-F determined in this study were similar to those against LDN and LDN-F measured by
ELISA as shown in the previous study (Eberl et al., 2001b). A slight difference was that during the vaccination we could not detect the initial anti-LDN IgM response (Fig. 1). The IgG response against LDN-F and LDN was much more variable within the two groups than that for IgM (data not shown), as seen by Eberl et al. (2001b). Notably, the total antibody response to LDN-F and LDN was lower than the total anti-carbohydrate antibody responses against LDN-DF,
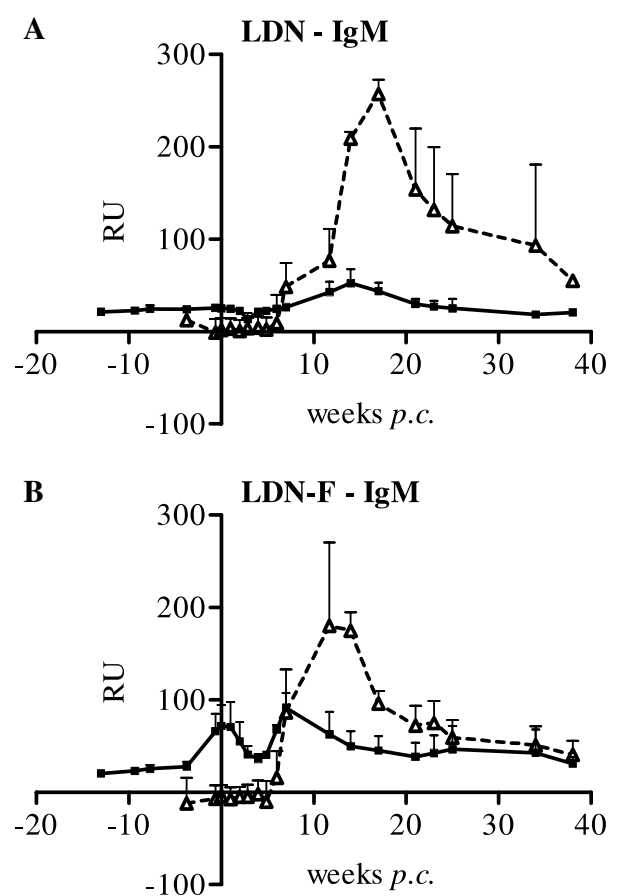

Fig. 1. Levels of carbohydrate-specific antibodies, shown as mean RUs and SEM of three chimpanzees for IgM antibodies against LDN (A) and LDN-F (B). Triangles: control group; squares: vaccinated group. 
F-LDN-F, and F-LDN. The antibodies detected against F-LDN and F-LDN-F showed identical binding profiles, indicating that the proximal fucose is not an important part of the main epitope, therefore only data obtained with F-LDN are shown.

In general, the total antibody levels to LDN, LDN-F, and LDN-DF were higher in controls compared to those of the vaccinated chimpanzees, while the antibody levels to F-LDN were similar in both groups. The total serum antibody levels to F-LDN and LDN-DF were at least three times higher than the antibody levels to LDN-F and LDN. In both groups, the antibodies specific for LDN-DF and F-LDN are predominantly IgGs. The IgM response to LDN-DF and F-LDN followed the IgG response but was $10 \%$ or even less compared to IgG (data not shown).

In the vaccinated group, F-LDN specific-IgG was present at the first sampling time point 3.5 weeks after the first immunization. The level of antibodies increased with each vaccination, peaked at the time of challenge infection and declined during 5 weeks before rising again. A secondary peak was shown at week 12 p.c. that slowly decayed (Fig. 2A). In the controls, the antibody response to F-LDN was not evident before week 5 p.c. and peaked between weeks 12 and 17 p.c., with maxi-
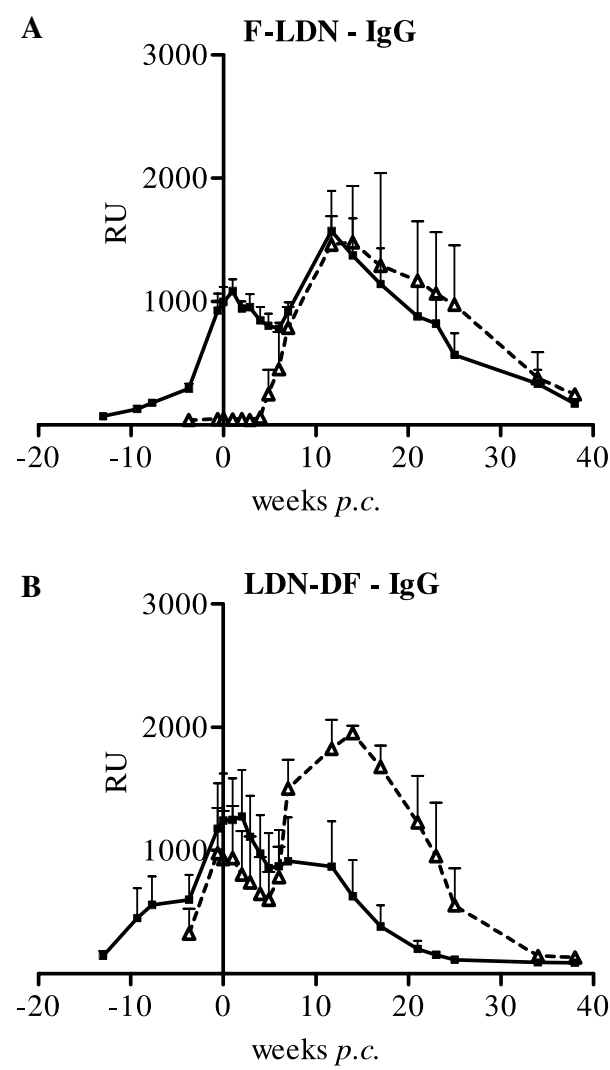

Fig. 2. Levels of carbohydrate-specific antibodies, shown as mean RUs and SEM of three chimpanzees for IgG antibodies against F-LDN (A) and LDN-DF (B). Triangles: control group; squares: vaccinated group.

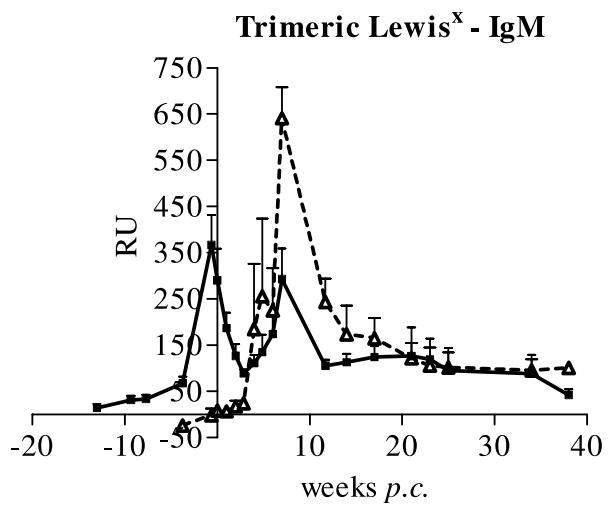

Fig. 3. Levels of carbohydrate-specific antibodies, shown as mean RUs and SEM of three chimpanzees for IgM antibodies against trimeric Lewis ${ }^{\mathrm{x}}$. Triangles: control group; squares: vaccinated group.

mum levels equal to those in the vaccinated animals (Fig. 2A).

In the vaccinated group, the LDN-DF specific-IgG rose gradually after vaccination, reached maximum levels at the time of challenge (similar to the F-LDN response), leveled off from week 5 p.c. till week 12 p.c. and thereafter returned near baseline (Fig. 2B). Surprisingly, in the controls the LDN-DF-specific IgG was already present at time of challenge, however levels returned after 36 weeks p.c. to baseline. A higher secondary peak from week 7 p.c. till week 17 p.c. was seen in the controls than in the vaccinated chimpanzees (Fig. 2B).

In addition, the antibody response to carbohydrate structures that are related to the schistosome circulating antigen CCA were studied. High levels of antibodies predominantly of the IgM isotype, were detected against trimeric Lewis ${ }^{\mathrm{x}}$, a CCA related structure. No antibodies of the IgG isotype could be detected against trimeric Lewis $^{\mathrm{x}}$. The vaccinated chimpanzees showed an increase of the trimeric Lewis ${ }^{\mathrm{x}}$ specific $\operatorname{IgM}$ after each vaccination peaking at challenge time with a second peak during the acute phase of infection (Fig. 3). The control infected animals showed an increase in IgM antibodies during the acute phase of infection after which the level of antibodies decreased and stabilized at $100 \mathrm{RU}$ (Fig. 3). In both groups of chimpanzees, the binding profile of the antibodies against dimeric Lewis ${ }^{\mathrm{x}}$ was similar compared to the antibody responses against trimeric Lewis ${ }^{\mathrm{x}}$, but the level was much lower (data not shown). The antibody response to monomeric Lewis ${ }^{\mathrm{x}}$ was negligible, which is consistent with the earlier study by Eberl et al. (2001b).

\section{Discussion}

In a longitudinal study we have monitored anti-carbohydrate responses in schistosome-exposed vaccinated 
and control chimpanzees during the acute and chronic phase of schistosomiasis. Previously, Eberl et al. (2001b) found that virtually the entire vaccine-induced antibody response in this group of chimpanzees was directed to periodate-sensitive glycan epitopes while levels of antibodies recognizing periodate-resitant epitopes became markedly positive only when the infection progressed from the acute to the chronic phase. It was also demonstrated that LDN and LDN-F and to a lesser extent LNFPIII (containing Lewis ${ }^{\mathrm{x}}$ ) were recognized (Eberl et al., 2001b). However, in earlier studies in infected humans we have shown that the levels of antibody responses to LDN, LDN-F, and Lewis ${ }^{\mathrm{x}}$, structures shared between schistosomes and the host, are lower than the responses to LDN-DF and F-LDN, structures that are more restricted to schistosomes and not shared between the parasite and the host (Naus et al., 2003; Van Remoortere et al., 2001). Therefore, we have now focused on the humoral immune response of chimpanzees to the latter structures.

Indeed, we found that the antibody levels to the LDN-DF, F-LDN, and trimeric Lewis ${ }^{\mathrm{x}}$ epitopes were strikingly higher than those against LDN, LDN-F, and monomeric Lewis ${ }^{\mathrm{x}}$, which is consistent with the earlier findings in humans (Naus et al., 2003; Van Remoortere et al., 2001). Interestingly, the anti-LDN-DF and antiF-LDN antibodies were predominantly IgGs, whereas anti-Lewis ${ }^{\mathrm{x}}$, anti-LDN, and anti-LDN-F were IgMs. It is relatively common that autoantibodies in sera of healthy individuals are predominantly of the $\operatorname{IgM}$ subclass (George and Shoenfield, 1996). IgG autoantibodies could be harmfull, because of their ability to induce antibody-dependent cellular cytotoxicity. It has been suggested that self-reactive B cells may possess control over Ig isotype switching (Phan et al., 2003). The antiLDN, LDN-F, and Lewis ${ }^{\mathrm{x}}$ epitopes are all expressed on a number of human glycoproteins and thus in view of the close genetic relationship presumably also on a number of chimpanzee glycoproteins. Probably the persistent presentation of these glycans or the underlying antigens that present these epitopes contributes to the induction of autoantibodies. The phenomenon that the IgG antibody response against LDN or LDN-F varied strongly between the animals, might be explained by the fact that this "auto-antibody" response is likely to differ more strongly from individual to individual than a response against non-self glycans. In earlier studies in infected humans we have shown a similar variation in the levels of IgGs to LDN and LDN-F (Naus et al., 2003; Van Remoortere et al., 2001).

Previously, it was shown that T-cell independent egg glycan antigens elicit antibody responses of the IgM and IgG2-type that are cross-reactive with antigens of schistosomula. These antibodies not only fail to mediate antibody-dependent cytotoxicity but at the same time may block effector antibodies like IgG1 and IgE (But- terworth et al., 1992; Dunne et al., 1987). Therefore, the anti-LDN, LDN-F, and Lewis ${ }^{\mathrm{x}} \operatorname{IgM}$ antibodies may act as blocking antibodies. However, this is not likely because of the low levels of IgMs in comparison with those of IgG. Recently, Naus et al. (2003) showed that in human infections the IgG antibodies directed against FLDN and LDN-DF were predominantly of the IgG1 isotype. We assume that the anti-F-LDN and LDN-DF IgG antibodies found in sera of chimpansees vaccinated or infected with $S$. mansoni were of the IgG1 isotype and therefore may play a role in cytotoxicity.

An additional aspect of this study concerns the development of antibody responses in vaccinated versus control chimpanzees. In general, the vaccination was partly effective as a $40 \%$ reduction in egg output was found in vaccinated chimpanzees compared with control chimpanzees (Eberl et al., 2001b). During the vaccination the antibody response was primarily directed against carbohydrate structures, which might imply that the anti-carbohydrate antibodies are involved in protection. Indeed, several older papers have already described that anti-carbohydrate antibodies are involved in protection to schistosome infections (Bickle et al., 1986; Gregoire et al., 1987), although the mechanisms behind this protection are largely unclear. In view of this, it would be interesting to study if schistosome-characteristic glycans such as F-LDN and LDN-DF could be regarded as vaccine candidates for human schistosomiasis.

As a general pattern, we showed that the vaccinated chimpanzees displayed lower anti-carbohydrate antibody levels than the control animals. This might indicate a memory-induced down-regulation of the anti-carbohydrate immune response after vaccination. Alternatively, since egg production was reported to be lower in the vaccinated animals, the lower anti-carbohydrate responses could possibly be due to the lower antigen levels as a result of the advantageous effect of vaccination (Eberl et al., 2001a). It should be mentioned, however, that in general antibody responses do not correlate with infection intensity.

In contrast to the other epitopes, the levels of anti-FLDN antibodies were similarly intense in both animal groups. Kantelhardt et al. (2002) demonstrated that Fucal-3GalNAc is a major antigenic motif of $S$. mansoni glycolipids and appeared to be the basis for the cross-reactivity with KLH. Eberl et al. (2001b) demonstrated both in vaccinated and infected chimpanzees high levels of IgGs against KLH. Those binding profiles are qualitatively comparable with the anti-F-LDN IgGs determined in this study. Previously it has been shown that antibodies recognizing a carbohydrate epitope that is cross-reactive with KLH have been associated with protection (Grzych et al., 1987), suggesting that this structure may be used for immunoprophylaxis.

The presence of antibodies directed against F-LDN and LDN-DF correspond with structural and 
immunocytochemical data on schistosome glycosylation of the different life-stages of the parasite (Khoo et al., 1997a,b, 1995; Van Remoortere et al., 2000; Wuhrer et al., 2002). Additionaly, the antibody reponses to LDN-DF and F-LDN were elicited during vaccination, suggesting that the irradiated cercariae express or secrete antigens that contain these epitopes. Remarkably, sera of two of the control chimpanzees already had $\operatorname{IgG}$ antibodies against the LDN-DF structure at time of challenge. Apparently the anti-LDN-DF antibodies were previously induced by other immunostimulatory factors e.g., food or previous infections, indicating that LDN-DF epitope is obviously not restricted to schistosomes only. However, the binding profile of anti-LDNDF antibodies had a similar pattern as anti-F-LDN antibodies and therefore followed the infection. In sera of humans which were not infected with $S$. mansoni we have never been able to detect anti-LDN-DF antibodies (Van Remoortere et al., 2001).

In addition to the fact that LDN-DF and F-LDN are important targets for antibodies, it was recently shown that glycoconjugates containing LDN-DF could stimulate innate cellular immune responses. Compared to glycoconjugates carrying Lewis ${ }^{\mathrm{x}}$, LDN or LDN-F structures, the LDN-DF conjugate was a superior inducer of IL-10 production in peripheral blood mononuclear cells (PBMCs) of naive human donors (Van der Kleij et al., 2002). With regard to Lewis ${ }^{\mathrm{x}}$, it has been demonstrated that the monomeric Lewis ${ }^{\mathrm{x}}$ antigens induce IL 10 production by PBMC of schistosome-infected individuals (Velupillai et al., 2000) and, in murine models skew immune responses toward Th2 responses (Okano et al., 2001).

The antibody responses directed against trimeric Lewis $^{\mathrm{x}}$ are exceptional with regard to intensity and isotype when compared with the rest of the epitopes tested. We found relative high levels of anti-trimeric Lewis ${ }^{\mathrm{x}} \operatorname{IgMs}$ but no IgGs. In general, either low levels of IgM antibodies were found against host-shared carbohydrate structures or relative high levels of IgG antibodies against schistosome-characteristic carbohydrate structures. The different response seen for antibodies directed against trimeric Lewis ${ }^{\mathrm{x}}$ may be caused by the multimericity of Lewis ${ }^{\mathrm{x}}$, which is currently under investigation.

In conclusion, both vaccination and infection of chimpanzees with cercariae of $S$. mansoni induce an initial anti-carbohydrate response. A consistent and much higher antibody response was found to the carbohydrate structures that are characteristic for schistosomes in comparison to those structures shared with the host. For future vaccination and infection studies it will be beneficial to analyze immune responses to schistosome-characteristic carbohydrate structures in addition to host-shared carbohydrate structures or to peptides.

\section{Acknowledgments}

We are grateful to Dr. B. Appelmelk for supplying the mono-, di-, and trimeric Lewis ${ }^{\mathrm{x}}$ HSA. This study was supported by the Netherlands Foundation for Chemical Research (CW) and Life Science Foundation (LW) with financial aid from the Netherlands Organization for Scientific Research (NWO).

\section{References}

Ágoston, K., Kérèkgyarto, J., Hajko, J., Batta, G., Lefeber, D.J., Kamerling, J.P., Vliegenthart, J.F., 2002. Synthesis of fragments of the glycocalyx glycan of the parasite Schistosoma mansoni. Chemistry $8,151-161$.

Berggren, W.L., Weller, T.H., 1967. Immunoelectrophoretic demonstration of specific circulating antigen in animals infected with Schistosoma mansoni. Am. J. Trop. Med. Hyg. 16, 606-612.

Bergwerff, A.A., Van Dam, G.J., Rotmans, J.P., Deelder, A.M., Kamerling, J.P., Vliegenthart, J.F., 1994. The immunologically reactive part of immunopurified circulating anodic antigen from Schistosoma mansoni is a threonine-linked polysaccharide consisting of $\rightarrow 6$-(beta-D-GlcpA-( $1 \rightarrow 3$ ))-beta-D-GalpNAc- $1 \rightarrow$ repeating units. J. Biol. Chem. 269, 31510-31517.

Bergwerff, A.A., Van Oostrum, J., Kamerling, J.P., Vliegenthart, J.F., 1995. The major N-linked carbohydrate chains from human urokinase. The occurrence of 4-O-sulfated, (alpha 2-6)-sialylated or (alpha 1-3)- fucosylated $N$-acetylgalactosamine(beta 1-4)- $N$ acetylglucosamine elements. Eur. J. Biochem. 228, 1009-1019.

Bickle, Q.D., Andrews, B.J., Taylor, M.G., 1986. Schistosoma mansoni: characterization of two protective monoclonal antibodies. Parasite Immunol. 8, 95-107.

Butterworth, A.E., Dunne, D.W., Fulford, A.J., Thorne, K.J., Gachuhi, K., Ouma, J.H., Strurrock, R.F., 1992. Human immunity to Schistosoma mansoni: observations and mechanisms, and implications for control. Immunol. Invest. 21, 391-407.

Cummings, R.D., Nyame, A.K., 1999. Schistosome glycoconjugates. Biochim. Biophys. Acta 1455, 363-374.

Deelder, A.M., Kornelis, D., Van Marck, E.A., Eveleigh, P.C., Van Egmond, J.G., 1980. Schistosoma mansoni: characterization of two circulating polysaccharide antigens and the immunological response to these antigens in mouse, hamster, and human infections. Exp. Parasitol. 50, 16-32.

Deelder, A.M., Van Dam, G.J., Kornelis, D., Fillie, Y.E., van Zeyl, R.J., 1996. Schistosoma: analysis of monoclonal antibodies reactive with the circulating antigens CAA and CCA. Parasitology 112, 2135 .

Dell, A., Morris, H.R., Easton, R.L., Panico, M., Patankar, M., Oehniger, S., Koistinen, R., Koistinen, H., Seppala, M., Clark, G.F., 1995. Structural analysis of the oligosaccharides derived from glycodelin, a human glycoprotein with potent immunosuppressive and contraceptive activities. J. Biol. Chem. 270, 24116-24126.

Dunne, D.W., Bickle, Q.D., Butterworth, A.E., Richardson, B.A., 1987. The blocking of human antibody-dependent, eosinophilmediated killing of Schistosoma mansoni schistosomula by monoclonal antibodies which cross-react with a polysaccharide-containing egg antigen. Parasitology 94, 269-280.

Eberl, M., Langermans, J.A., Frost, P.A., Vervenne, R.A., Van Dam, G.J., Deelder, A.M., Thomas, A.W., Coulson, P.S., Wilson, R.A., 2001a. Cellular and humoral immune responses and protection against schistosomes induced by a radiation-attenuated vaccine in chimpanzees. Infect. Immun. 69, 5352-5362.

Eberl, M., Langermans, J.A., Vervenne, R.A., Nyame, A.K., Cummings, R.D., Thomas, A.W., Coulson, P.S., Wilson, R.A., 2001 b. 
Antibodies to glycans dominate the host response to schistosome larvae and eggs: is their role protective or subversive? J. Infect. Dis. $183,1238-1247$.

Fox, N., Damjanov, I., Knowles, B.B., Solter, D., 1983. Immunohistochemical localization of the mouse stage-specific embryonic antigen 1 in human tissues and tumors. Cancer Res. 43, 669-678.

Fukuda, M.N., Dell, A., Oates, J.E., Wu, P., Klock, J.C., Fukuda, M., 1985. Structures of glycosphingolipids isolated from human granulocytes. The presence of a series of linear poly- $N$-acetyllactosaminylceramide and its significance in glycolipids of whole blood cells. J. Biol. Chem. 260, 1067-1082.

George, J., Shoenfield, Y., 1996. Natural autoantibodies. In: Peter, J.B., Shoenfield, Y. (Eds.), Autoantibodies. Elsevier, Amsterdam, pp. 534-539.

Gregoire, R.J., Shi, M.J., Rekosh, D.M., Loverde, P.T., 1987. Protective monoclonal antibodies from mice vaccinated or chronically infected with Schistosoma mansoni that recognize the same antigens. J. Immunol. 139, 3792-3801.

Grzych, J.M., Dissous, C., Capron, M., Torres, S., Lambert, P.H., Capron, A., 1987. Schistosoma mansoni shares a protective carbohydrate epitope with keyhole limpet hemocyanin. J. Exp. Med. 165, 865-878.

Hakomori, S., Nudelman, E., Levery, S., Solter, D., Knowles, B.B., 1981. The hapten structure of a developmentally regulated glycolipid antigen (SSEA-1) isolated from human erythrocytes and adenocarcinoma: a preliminary note. Biochem. Biophys. Res. Commun. 100, 1578-1586.

Halkes, K.M., Lefeber, D.J., Fransen, C.T., Kamerling, J.P., Vliegenthart, J.F., 1998. Synthesis of the spacer-containing beta-DGalpNAc- $(1 \rightarrow 4)$-beta-D- GlcpNAc- $(1 \rightarrow 3)$-alpha-D-Galp moiety, representing the non-fucosylated backbone trisaccharide of the glycocalyx glycan of the parasite Schistosoma mansoni. Carbohydr. Res. 308, 329-338.

Hokke, C.H., Deelder, A.M., 2001. Schistosome glycoconjugates in host-parasite-interplay. Glycoconj. J. 18, 573-587.

Kantelhardt, S.R., Wuhrer, M., Dennis, R.D., Doenhoff, M.J., Bickle, Q., Geyer, R., 2002. Fuc(alpha1 $\rightarrow$ 3)GalNAc-: major antigenic motif of Schistosoma mansoni glycolipids implicated in infection sera and keyhole limpet hemocyanin cross-reactivity. Biochem. J. $366,217-223$.

Khoo, K.H., Chatterjee, D., Caulfield, J.P., Morris, H.R., Dell, A., 1997a. Structural characterization of glycophingolipids from the eggs of Schistosoma mansoni and Schistosoma japonicum. Glycobiology 7, 653-661.

Khoo, K.H., Chatterjee, D., Caulfield, J.P., Morris, H.R., Dell, A., 1997b. Structural mapping of the glycans from the egg glycoproteins of Schistosoma mansoni and Schistosoma japonicum: identification of novel core structures and terminal sequences. Glycobiology 7, 663-677.

Khoo, K.H., Sarda, S., Xu, X., Caulfield, J.P., McNeil, M.R., Homans, S.W., Morris, H.R., Dell, A., 1995. A unique multifucosylated -3 GalNAc beta $1 \rightarrow 4$ GlcNAc beta $1 \rightarrow 3$ Gal alpha 1 motif constitutes the repeating unit of the complex $O$-glycans derived from the cercarial glycocalyx of Schistosoma mansoni. J. Biol. Chem. 270, 17114-17123.

Ko, A.I., Drager, U.C., Harn, D.A., 1990. A Schistosoma mansoni epitope recognized by a protective monoclonal antibody is identical to the stage-specific embryonic antigen 1. Proc. Natl. Acad. Sci. USA $87,4159-4163$.

Morris, H.R., Dell, A., Easton, R.L., Panico, M., Koistinen, H., Koistinen, R., Oehninger, S., Patankar, M.S., Seppala, M., Clark, G.F., 1996. Gender-specific glycosylation of human glycodelin affects its contraceptive activity. J. Biol. Chem. 271, 32159-32167.

Naus, C.W.A., Van Remoortere, A., Ouma, J.H., Kimani, G., Dunne, A.W., Kamerling, J.P., Deelder, A.M., Hokke, C.H., 2003. Specific antibody responses to three schistosome-related carbohydrate structures in recently exposed immigrants and established residents in an area of Schistosoma mansoni endemicity. Infect. Immun. 71, $5676-5681$.

Okano, M., Satoskar, A.R., Nishizaki, K., Harn Jr, D.A., 2001. Lacto$N$-fucopentaose III found on Schitosoma mansoni egg antigens functions as adjuvant for proteins by inducing Th2-type response. J. Immunol. 167, 442-450.

Omer-Ali, P., Mansour, M., Woody, J.N., Smithers, S.R., Simpson, A.J., 1989. Antibody to carbohydrate and polypeptide epitopes on the surface of schistosomula of Schistosoma mansoni in Egyptian patients with acute and chronic schistosomiasis. Parasitology 98 (Pt. 3), 417-424.

Phan, T.G., Amesbury, M., Gardam, S., Crosbie, J., Hasbold, J., Hodgkin, P.D., Basten, A., Brink, R., 2003. B cell receptorindependent stimuli trigger immunoglobulin class switch recombination and production of $\operatorname{IgG}$ autoantibodies by anergic selfreactive B cells. J. Exp. Med. 259, 845-860.

Spooncer, E., Fukuda, M., Klock, J.C., Oates, J.E., Dell, A., 1984. Isolation and characterization of polyfucosylated lactosaminoglycan from human granulocytes. J. Biol. Chem. 259, 4792-4801.

Van Dam, G.J., Bergwerff, A.A., Thomas-Oates, J.E., Rotmans, J.P., Kamerling, J.P., Vliegenthart, J.F., Deelder, A.M., 1994. The immunologically reactive O-linked polysaccharide chains derived from circulating cathodic antigen isolated from the human blood fluke Schistosoma mansoni have Lewis x as repeating unit. Eur. J. Biochem. 225, 467-482.

Van den Eijnden, D.H., Neeleman, A.P., Van der Knaap, W.P., Bakker, H., Agterberg, M., Van Die, I., 1995. Novel glycosylation routes for glycoproteins: the lacdiNAc pathway. Biochem. Soc. Trans. 23, 175-179.

Van der Kleij, D., Van Remoortere, A., Schuitemaker, J.N.H., Kapsenberg, M.L., Deelder, A.M., Tielens, A.G., Hokke, C.H., Yazdanbakhsh, M., 2002. Triggering of innate immune responses by schistosome egg glycolipids and their carbohydrate epitope GalNAc $\beta 1-4$ (Fuc $\alpha 1-2$ Fuc $\alpha 1-3)$ GlcNAc. J. Infect. Dis. 185, 531539.

Van Kuik, J.A., de Waard, P., Vliegenthart, J.F., Klein, A., Carnoy, C., Lamblin, G., Roussel, P., 1991. Isolation and structural characterization of novel neutral oligosaccharide-alditols from respiratory-mucus glycoproteins of a patient suffering from bronchiectasis. 2. Structure of twelve hepta-to- nonasaccharides, six of which possess the GlcNAc beta(1-3)[Gal beta(1-4)GlcNAc beta(16)] Gal beta(1-3)GalNAc-ol common structural element. Eur. J. Biochem. 198, 169-182.

Van Remoortere, A., Hokke, C.H., Van Dam, G.J., Van Die, I., Deelder, A.M., Van den Eijnden, D.H., 2000. Various stages of schistosoma express Lewis(x), LacdiNAc, GalNAcbeta1-4 (Fucalpha1-3)GlcNAc and GalNAcbeta1-4(Fucalpha1-2Fucalpha13)GlcNAc carbohydrate epitopes: detection with monoclonal antibodies that are characterized by enzymatically synthesized neoglycoproteins. Glycobiology 10, 601-609.

Van Remoortere, A., Van Dam, G.J., Hokke, C.H., Van den Eijnden, D.H., Van Die, I., Deelder, A.M., 2001. Profiles of immunoglobulin $\mathrm{M}(\mathrm{IgM})$ and $\operatorname{IgG}$ antibodies against defined carbohydrate epitopes in sera of Schistosoma-infected individuals determined by surface plasmon resonance. Infect. Immun. 69, 2396-2401.

Velupillai, P., dos Reis, E.A., dos Reis, M.G., Harn, D.A., 2000. Lewis(x)-containing oligosaccharide attenuates schistosome egg antigen-induced immune depression in human schistosomiasis. Hum. Immunol. 61, 225-232.

Wuhrer, M., Kantelhardt, S.R., Dennis, R.D., Doenhoff, M.J., Lochnit, G., Geyer, R., 2002. Characterization of glycosphingolipids from Schistosoma mansoni eggs carrying Fuc(alpha1-3)GalNAc-, GalNAc(beta1-4)[Fuc(alpha1-3)]GlcNAc- and Gal(beta1-4)[Fuc(alpha1-3)]Gl. Eur. J. Biochem. 269, 481-493. 